\title{
Meningococcemia Complicada por Miocardite
}

\author{
Francisco Manes A lbanesi Fo, Ricardo Mourilhe Rocha, Rui Alves Barros
}

Rio de Janeiro, RJ

\begin{abstract}
Homem de 26 anos com meningococcemia (meningite), complicada por miocardite (disfunção ventricular e isquemia miocárdica), requereu tratamento para insuficiência cardíaca e evolui com regressão da disfunção miocárdica, seis meses após o quadro infeccioso.
\end{abstract}

\section{Meningococcemia Complicated by Myocarditis}

We report a case of a 26-year old man with meningococcemia complicated with myocarditis (ventricular dysfunction and myocardial ischemia), that required treatment for heart failure. Regression of myocardial dysfunction was observed six months after the infection.
A meningococcemia é uma síndrome infecciosa causada pelo diplococcus gram negativo, a neisseria meningitidis, germe que tem o homem como o seu reservatório natural, habitando e colonizando o nasofaringe ${ }^{1}$. Nos casos de infeção desta região, o germe pode penetrar na corrente sangüínea e ganhar todo o organismo ${ }^{1}$. A mais freqüente síndrome clínica desencadeada pela meningococcemia é a meningite aguda purulenta ${ }^{2}$, que representa em nosso meio importante problema de saúde pública, sendo responsável por inúmeros casos, ocorridos principalmente, no outono e no inverno, mesmo nos grandes centros urbanos.

Estimou-se que tenham ocorrido, entre 1939 a 1962, 600.000 casos de doença meningocócica em todo o mundo, com mais de 100.000 óbitos, a grande maioria em crianças ${ }^{2}$.

Como o germe se difunde por todo o organismo, ele pode ocasionar complicações infecciosas, além da meningite, sendo a mais encontrada a artrite supurativa, vindo a seguir a panoftalmite e o envolvimento cardíaco exteriorizado, mais freqüentemente, pela pericardite purulenta ${ }^{3}$. O comprometimento pericárdico é pouco freqüente e, geralmente, sem manifestações clínicas, o que torna difícil sua caracterização ${ }^{3}$. O acometimento miocárdico é raro ${ }^{4-6}$, sendo a sua grande maioria relatada como achado de necropsia ${ }^{7-9}$. Este trabalho tem por objetivo relatar caso de meningococcemia exteriorizado por meningite, com comprometimento miocárdico, ocorrido no fim do inverno no Rio de Janeiro.

Serviço de Cardiologia da Universidade do Estado do Rio de Janeiro Correspondência: Francisco Manes Albanesi F $F^{\circ}$ Av. 28 de Setembro 77 - $2^{\circ}$ 20551-030 - Rio de Janeiro, RJ

Recebido para publicação em 26/9/97

Aceito em 6/11/97

\section{Relato do Caso}

Homem de 26 anos, pardo, morador no município de Duque de Caxias (RJ), foi internado em 17/09/96 no hospital de isolamento do Estado do Rio de Janeiro, por apresentar quadro de meningococcemia, com petéquias, rash cutâneo e meningite. As petéquias foram observadas nas regiões plantar direita, palmar esquerda e nas extremidades dos membros inferiores, com áreas de coalescência, formando lesões de aparência equimótica. A punção lombar possibilitou o isolamento do meningococcus e sua tipificação no sorogrupo $\mathrm{C}$, tendo sido tratado com penicilina por 10 dias. O paciente, previamente, não apresentava sintomas cardiovasculares e praticava esportes sem nenhuma restrição. Durante a hospitalização foi observado sopro sistólico ao nível do $3^{\circ}-4^{\circ}$ espaços intercostais esquerdo, e sugerido que após a alta procurasse tratamento especializado na Cardiologia. Em 8/10/96 foi visto no Setor de Cardiomiopatias do HUPE com o seguinte quadro: dispnéia aos grandes e médios esforços, tosse sem expectoração e febre de 38 a $38,4^{\circ} \mathrm{C}$. Ao exame, apresentava turgência venosa +/ 4, pressão arterial de $104 / 72 \mathrm{mmHg}$, frequiência cardíaca de 56bpm., presença de $3^{\mathrm{a}}$ bulha na ponta, sopro sistólico $++/ 4$ na ponta e diminuição do murmúrio vesicular na base do pulmão direito. O eletrocardiograma (ECG) mostrava isquemia subepicárdica da parede ínfero-ântero-lateral (fig. 1) e a teleradiografia de tórax evidenciava cardiomegalia ++ / 4 com aumento das câmaras cardíacas, com derrame pleural à direita (fig. 2). O ecocardiograma revelava átrio esquerdo (AE) de 4,3cm; diâmetro sistólico do ventrículo esquerdo (DSVE) de 5,0cm; diâmetro diastólico do ventrículo esquerdo (DDVE) de 6,5cm; septo interventricular (SIV) de 1,0cm; parede posterior do ventrículo esquerdo (ppVE) de $1,1 \mathrm{~cm}$; 


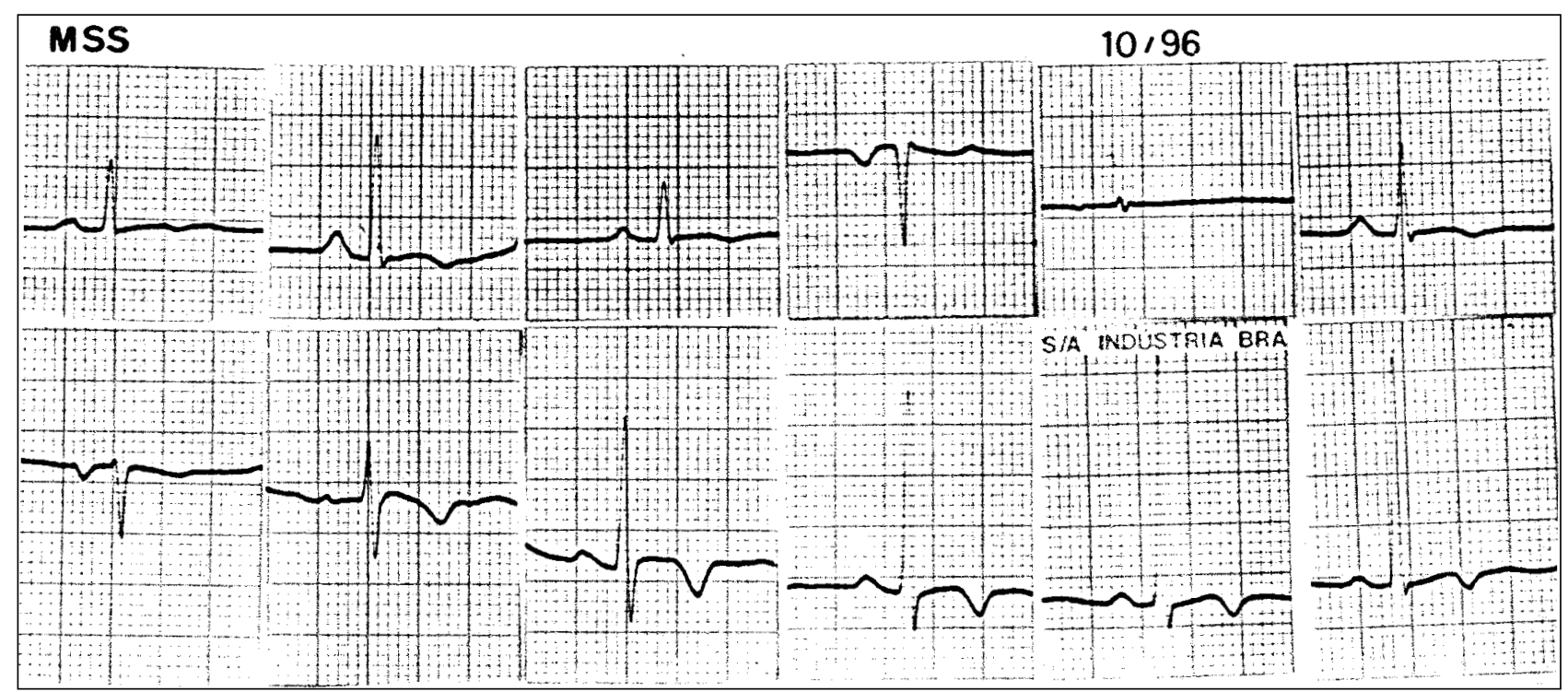

Fig. 1 - Eletrocardiograma de outubro/96 mostrando ritmo sinusal e isquemia subepicárdica ínfero-ântero-lateral.

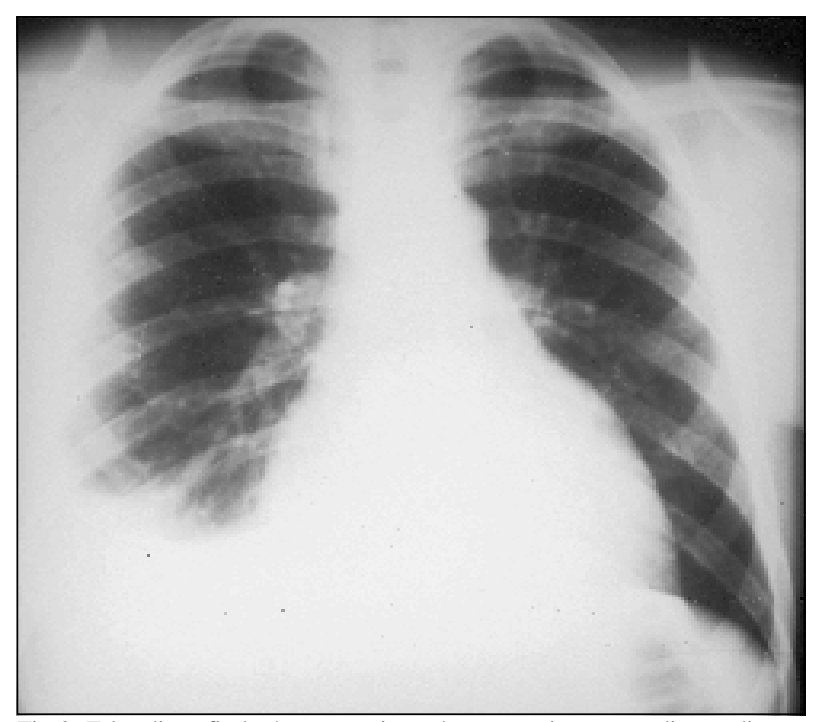

Fig. 2 - Teleradiografia de tórax em posição póstero-anterior, com cardiomegalia, por aumento do arco inferior esquerdo (ventrículo esquerdo) e derrame pleural à direta. diâmetro do ventrículo direito de $2,4 \mathrm{~cm}$; fração de ejeção de $45 \%$; hipocinesia difusa de grau leve, valvas sem espessamentos, presença de regurgitação mitral e tricúspide de graus leve (+/4). Foi medicado com digital (digoxina $0,25 \mathrm{mg} / \mathrm{dia}$ ), furosemida ( $80 \mathrm{mg} / \mathrm{dia})$ e inibidor da enzima conversora de angiotensina (lisinopril - 10mg/dia). Em 15 dias estava afebril, com diminuição da dispnéia e da tosse, com desaparecimento da $3^{\mathrm{a}}$ bulha e da síndrome de derrame pleural à direita. Foi reduzido o diurético para $40 \mathrm{mg} / \mathrm{dia}$. $\mathrm{O}$ ECG apresentou normalização das alterações isquêmicas em 90 dias (fig. 3). Após seis meses do quadro infeccioso inicial, estava assintomático, a teleradiografia de tórax mostrava apenas discreto aumento do ventrículo esquerdo, o ecocardiograma demonstrava: DDVE de 6,1 cm; DSVE de $3,8 \mathrm{~cm}$; AEde 3,9cm; SIV de 0,6cm; ppVEde 0,7cm; fração de ejeção de $74 \%$. A medicação foi redimensionada, sendo suspenso o cardiotônico e, posteriormente, o diurético, estando hoje um ano após a meningococcemia, apenas em uso de inibidor da enzima conversora de angiotensina .

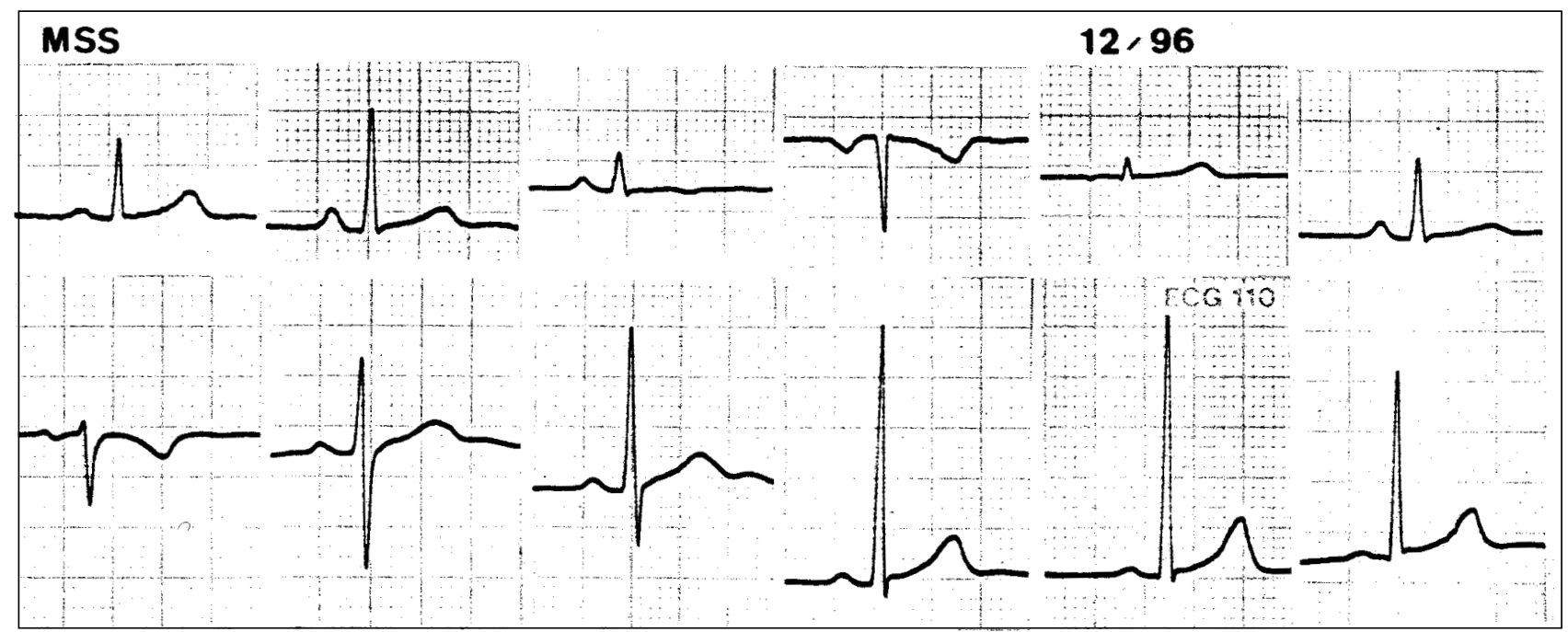

Fig. 3 - Eletrocardiograma de dezembro/96 com normalização da repolarização ventricular. 


\section{Discussão}

A doença meningocócica contínua representando importante causa de morbidade e mortalidade, mesmo em países desenvolvidos como os Estados Unidos da América (EUA), ocasionando 3000 casos/ano, acometendo, na maioria, crianças e adultos jovens, sendo, atualmente, mais encontrados os sorogrupo $\mathrm{B}$ e $\mathrm{C}^{10-12}$, porém, nos últimos anos têm sido relatados casos resistentes ao tratamento com a penicilina ${ }^{13}$. Nosso paciente era adulto jovem, de 26 anos e que apresentou quadro de meningite por N.meningitidis do sorogrupo $\mathrm{C}$, respondendo bem ao tratamento com a penicilina, porém, iniciando os sintomas cardiovasculares durante a internação da meningite, sem no entanto, ter sido adequadamente esclarecido o motivo esse comprometimento.

No Rio de Janeiro, a meningococcemia é frequiente, sendo encontrada tanto no município do Rio de Janeiro, quanto no interior. Nosso paciente é oriundo da baixada fluminense (Grande Rio), teve a infeção no período do inverno, onde é mais prevalente a infeção por N.meningitidis, mesmo no estado onde o inverno não é rigoroso e sem definição marcada entre as estações climáticas. Entretanto, não encontramos relatos referindo esse acometimento miocárdico no estado, seria por não ter sido adequadamente procurado, pois, o sorogrupo C é encontrado com freqüência na região. O sorogrupo C (47\%) foi o mais encontrado entre 132 casos de doença meningocócica vista em Atlanta, tendo sido observado em $53 \%$ dos pacientes com meningite e em 83,33\% (10/12) dos casos com maiores seqüelas ${ }^{19}$. Em contraste com aqueles que desenvolveram bacteremia sem sepse, onde os sorogrupos B, Y e W135 foram observados em $80 \%$ dos $\operatorname{casos}^{14}$.

O acometimento cardíaco na meningococcemia é controverso; a maioria dos casos relatados é de pacientes com curso clínico grave, que evoluíram para o óbito, detectando-se à necropsia, miocardite entre $22 \mathrm{a} 78 \%{ }^{7-9}$ e pericardite em $4,3 \%{ }^{15}$.

O comprometimento miocárdico é visto com grande freqüência na necropsia, onde diferentes graus de miocardite (focal/difusa) são encontrados ${ }^{7,9}$, porém na clínica ele é pouco achado, somente quando os sinais e sintomas da insuficiência cardíaca são observados e, adequadamente, identificados ${ }^{16,17}$. Boucek e col ${ }^{4}$ demonstraram que a disfunção miocárdica em crianças não era diagnosticada na meningococcemia aguda não fatal, entretanto, quando encontrada, tinha grande significado prognóstico, pois em 42,85\% (3/7) das crianças com esse diagnóstico, foram a óbito.

Nosso paciente, adulto jovem (26 anos), sem antecedentes de doença prévia, porém, complicada com miocardite, respondeu bem ao tratamento com a penicilina, iniciando os sintomas cardiovasculares durante sua internação. Apesar de não ter sido, adequadamente, esclarecido o motivo desse comprometimento, teve boa evolução clínica. Em contraste com os dois casos em jovens de 19 anos vistos na África do Sul, também sem doença cardíaca prévia, que evoluíram com miocardite, sendo que um deles com episódios intermitentes de fibrilação atrial e extra-sistolia ventricular freqüente, faleceu com miocardite confirmadaà necropsia ${ }^{6}$.
Na epidemia ocorrida entre 1968-1969 na Base Área de Keesler, os 10 pacientes com meningococcemia, tinham infeção pelo sorogrupo C, sendo seis com meningite ${ }^{18}$. A insuficiência cardíaca congestiva foi encontrada em sete $(70 \%)$, todos homens jovens e sem antecedentes de cardiopatia prévia. Desses, três tinham radiografias de tórax com edema pulmonar, três apresentavam elevação da pressão venosa central (>12 cm $\left.\mathrm{H}_{2} \mathrm{O}\right)$ e um com galope ventricular que não estava presente na época da internação ${ }^{18}$. Todos responderam de modo satisfatório ao tratamento com restrição líquida e digital. Entre os pacientes, cinco apresentavam alterações eletrocardiográficas no segmento ST-T, sendo três dos sete com insuficiência cardíaca e dois dos três sem aquela síndrome, que regrediram após compensação do quadro.

Em 1987 Brasier e col ${ }^{5}$ descreveram um caso complicado por miopericardite, responsável pela apresentação da meningococcemia, antes do quadro de meningite, ocasionada pelo sorogrupo W 135 que, esporadicamente, manifestada na área geográfica de Boston nos EUA.

A presença de edema pulmonar no transcurso da meningococcemia poderia ser devido a pneumonia ou a lesão vascular secundária a liberação de endotoxinas ${ }^{18}$. A injeção de endotoxinas extraídas do meningococcus, dentro dos ventrículos laterais de cães, provoca o aparecimento de alterações miocárdicas e pulmonares, levantando a hipótese tóxica desse comprometimento ${ }^{19}$. Robboy ${ }^{20}$ relatou caso de homem de 22 anos, internado com meningite meningocócica, que evoluiu com insuficiências cardíaca e renal, além de coagulação vascular disseminada. O quadro da necrose tubular aguda, o distúrbio da coagulação e a meningite foram resolvidos, porém a disfunção miocárdica evoluiu progressivamente, com elevação da LDH, cardiomegalia e taquicardia persistente, até ao quadro de morte súbita. Na necropsia, o coração pesava $560 \mathrm{~g}$ com dilatação das quatro cavidades, porém, sem áreas de infiltração e necrose, somente sendo observadas áreas de atrofia e fibrose. No entanto, a análise do sistema de condução mostrou a presença de focos hemorrágicos e supuração na área do nó atrioventricular, tendo sido atribuída, como causa morte, a provável ocorrência de bloqueio atrioventricular total.

Devemos ficar atentos nos casos de meningococcemia, quando os pacientes apresentarem dispnéia, hipotensão e cianose, associadas a presença de taquicardia, galope e congestão pulmonar, para procedermos melhor avaliação do comprometimento miocárdico. OECG poderá ser de valia revelando alterações do segmento ST-T, porém, na maioria dos casos, ele só serve para pôr em evidência arritmias (incluindo taquicardia sinusal, supraventricular e ventricular), porém o mais observado é a presença de alterações inespecíficas da repolarização. A elevação dos níveis das enzimas cardíacas pode ser útil na confirmação do diagnóstico.

Na meningococcemia, a presença de hipotensão e choque é classicamente interpretada como decorrente da hemorragia nas supra renais, e conhecida com síndrome de Waterhouse- Friderischsen. Porém, na maioria dos casos de 
choque, as glândulas supra renais estão normais, sendo o choque de natureza cardiogênica devido à miocardite, segundo Sandler e col ${ }^{6}$.

Finalmente, devemos realçar a necessidade do exame cardiovascular mais cuidadoso nos pacientes com meningococcemia, a fim de não deixar o acometimento desse sistema passar despercebido nos pacientes com quadro infeccioso por N.meningitidis.

\section{Referências}

1. Greenfield S, Sheehe PR, Feldman HA - Meningococcal carriage in population of "normal" families. J Infect Dis 1971; 123: 67-73.

2. Feldman HA - Meningococcal infections. Adv Intern Med 1972; 18; 117-40.

3. Apicella MA-Meningococcal infections. In: Bennett JC, Plum F, ed-Cecil Textbook of Medicine. $20^{\text {th }}$ ed. Philadelphia: WS Saunders Co, 1996: 1618-25.

4. Boucek MM, Boerth RC, Artman M et al - Myocardial dysfunction in children with acute meningococcemia. J Pediatr 1984; 105: 538-42.

5. Brasier AR, Macklis JD, Vaughan D, Warner L, Kirshenbaum JM - Myopericarditis as an initial presentation of meningococcemia, unusual manifestation of infection with serotype W 135. Am J Med 1987; 82: 641-4.

6. Sandler MA, Pincus PS, Weltman MD et al-Meningococcaemia complicated by myocarditis. A report of 2 cases. S Afr Med J 1989; 75: 391-3.

7. Gore I, Saphier O - Myocarditis: a classification of 1042 cases. Am Heart J 1947; 34: $827-31$

8. Daniels WB - Cause of death in meningococcic infections; analysis of 300 cases. Am J Med 1950; 8: 468-73.

9. Hardman JM, Eark KM-Myocarditis in 200 fatal meningococcal infections. Arch Pathol 1969; 87: 318-25.

10. Jackson LA, Wenger JD - Laboratory-based surveillance for meningococcal disease in selected areas, United States, 1989-1991. MMWR CDC Surveill Summ. 1993; 42: 21-30.
11. Jackson LA, Schuchat A, Reeves MW, Wenger JD-Serogroup C meningococcal outbreaks in United States: epidemiology of an emerging threat. JAMA 1995; 273: 383-9.

12. Serogroup B meningococcal disease-Oregon, 1994. MMWR Morb Mortal Wky Rep 1995; 44: 121-4.

13. Woods CR, Smith AL, Wasilauskas BL, Campos J, Givner LB - Invasive disease caused by Neisseria meningitidis relatively resistant to penicillin in North Carolina. J Infect Dis 1994; 170: 453-6.

14. Herrick WW-Meningococcic pericarditis, with report of 12 cases. Med Clin Nort Am 1918; 2: 411-26.

15. Levin S, Painter MB - The treatment of acute meningococcal infection in adults. Ann Intern Med 1966; 64: 1049-56.

16. Kanter DM, Mauriello DA, Learner N - Acute meningococcemia with vascular collapse: An analysis of 10 recently treated cases. Am J Med Sci 1956; 232: 67487.

17. Denmark TC, Knight EL - Cardiovascular and coagulation complications of group C meningococcal disease. Arch Inter Med 1971; 127: 238-40.

18. Ducker TB, Simmons RL - The pathogenesis of meningitis. Arch Neurol 1968; 18: $123-8$.

19. Robboy SJ - Atrioventricular node inflammation - mechanism of sudden death in protracted meningococcemia. N Engl J Med 1972; 286: 1091-3. 